\title{
Ophthalmic manifestations of Heimler syndrome due to PEX6 mutations.
}

\author{
Nutsuchar Wangtiraumnuay \\ Wills Eye Hospital; Queen Sirikit National Institute of Child Health \\ Waleed Abed Alnabi \\ Wills Eye Hospital \\ Mai Tsukikawa \\ Thomas Jefferson University
}

Avrey Thau

Wills Eye Hosptial; Thomas Jefferson University

Jenina Capasso

Wills Eye Hospital

Follow this and additional works at: https://jdc.jefferson.edu/willsfp

Part of the Ophthalmology Commons

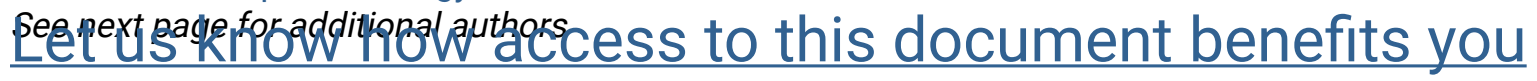

\section{Recommended Citation}

Wangtiraumnuay, Nutsuchar; Alnabi, Waleed Abed; Tsukikawa, Mai; Thau, Avrey; Capasso, Jenina; Sharony, Reuven; Inglehearn, Chris F.; and Levin, Alex V., "Ophthalmic manifestations of Heimler syndrome due to PEX6 mutations." (2018). Wills Eye Hospital Papers. Paper 83. https://jdc.jefferson.edu/willsfp/83

This Article is brought to you for free and open access by the Jefferson Digital Commons. The Jefferson Digital Commons is a service of Thomas Jefferson University's Center for Teaching and Learning (CTL). The Commons is a showcase for Jefferson books and journals, peer-reviewed scholarly publications, unique historical collections from the University archives, and teaching tools. The Jefferson Digital Commons allows researchers and interested readers anywhere in the world to learn about and keep up to date with Jefferson scholarship. This article has been accepted for inclusion in Wills Eye Hospital Papers by an authorized administrator of the Jefferson Digital Commons. For more information, please contact: JeffersonDigitalCommons@jefferson.edu. 
Authors

Nutsuchar Wangtiraumnuay, Waleed Abed Alnabi, Mai Tsukikawa, Avrey Thau, Jenina Capasso, Reuven Sharony, Chris F. Inglehearn, and Alex V. Levin 


\section{Ophthalmic Manifestations of Heimler Syndrome due to PEX6 Mutations}

Nutsuchar Wangtiraumnuay, MD, ${ }^{1,2}$ Waleed Abed Alnabi, MD, ${ }^{1}$ Mai Tsukikawa, ${ }^{3}$ Avrey Thau, MD, ${ }^{3}$ Jenina Capasso, $\mathrm{LCGC}^{1}{ }^{1}$ Reuven Sharony, $\mathrm{MD},{ }^{4}$ Chris F Inglehearn, $\mathrm{PhD},{ }^{5}$ Alex V Levin, MD, MHSc, ${ }^{1,3}$

${ }^{1}$ Wills Eye Hospital, Philadelphia, Pennsylvania, USA.

${ }^{2}$ Department of Ophthalmology; Queen Sirikit National Institute of Child Health, Bangkok, Thailand.

${ }^{3}$ Sidney Kimmel Medical College at Thomas Jefferson University, Philadelphia, Pennsylvania; USA.

${ }^{4}$ The Genetic Institute and Obstetrics and Gynecology Department, Meir Medical Center affiliated with the Sackler Faculty of Medicine, Tel Aviv University, Kfar Saba, Israel.

${ }^{5}$ Leeds Institute of Biomedical and Clinical Sciences, St. James's University Hospital, University of Leeds, Leeds LS9 7TF, UK.

Address for correspondence:

Alex V. Levin, MD, MHSc

Chief, Pediatric Ophthalmology and Ocular Genetics

Wills Eye Hospital, Suite 1210, 840 Walnut Street, Philadelphia, PA 19107-5109

Phone: 215-928-3418; Fax: 215-928-3983, Email: alevin@willseye.org

Running head: Macular dystrophy in Heimler Syndrome 


\section{Abstract}

Background/Aims: Pigmentary retinal dystrophy and macular dystrophy have been previously reported in Heimler syndrome due to mutations in PEX1. Here we reported the ocular manifestations in Heimler syndrome due to mutations in PEX6.

Materials and methods: Medical records were reviewed to identify patient demographics, ophthalmic and systemic findings, and results of diagnostic testing including whole genome sequencing

Results: Patient 1 is 12 -year-old boy with a novel mutation c.275T>G (p.Val92Gly) and known mutation c.1802G>A (p.Arg601GIn) in PEX6. Patient 2 is a 7 year- old girl with the same known c.1802G>A (p.Arg601GIn) mutation and another novel missense mutation c.296G>T (p.Arg99Leu). Both patients exhibited a pigmentary retinopathy. Visual acuity in patient 1 was 20/80 and 20/25 following treatment of intraretinal cystoid spaces with carbonic anhydrase inhibitors, while patient 2 had visual acuity of 20/20 in both eyes without intraretinal cysts. Fundus autofluorescence showed a multitude of hyperfluorescent deposits in the paramacular area of both eyes. OCTs revealed significant depletion of photoreceptors in both patients and macular intraretinal cystoid spaces in one patient. Full field electroretinograms showed normal or abnormal photopic but normal scotopic responses. Multifocal electroretinograms were abnormal.

Conclusions: Heimler syndrome due to biallelic PEX6 mutations demonstrates a macular dystrophy with characteristic fundus autofluorescence and may be complicated by intraretinal cystoid spaces.

Keyword: Heimler syndrome, PEX6, Macular dystrophy 


\section{Introduction}

Heimler syndrome was first described by Heimler et al. in $1991 .^{1}$ This autosomal recessive disorder has since been shown to be caused by biallelic mutations in PEX1 or PEX6. The cardinal features include acquired sensorineural hearing loss (SNHL), amelogenesis imperfecta of the teeth, retinal dystrophy and abnormal nails (punctate leukonychia or Beau's lines).

Pigmentary retinal dystrophy and macular dystrophy have been previously reported in Heimler syndrome. ${ }^{2-5}$ These prior reports primarily describe the ocular manifestations of Heimler syndrome due to mutations in PEX1. We previously reported a brief description of the ocular manifestations of one patient with Heimler syndrome due to mutations in PEX6, ${ }^{5}$ and now provide more detail about that patient and another.

\section{Materials and methods:}

This study is an observational, retrospective study. The medical records of all patients with the clinical diagnosis of Heimler syndrome seen at the Ocular Genetics Clinic at Wills Eye Hospital from 2010 to 2016 were reviewed retrospectively. IRB/Ethics Committee ruled that approval was not required for this study. Two patients with the diagnosis of Heimler syndrome were identified. Their medical records were reviewed to identify patient demographics, ophthalmic and systemic findings, and results of other testing. The patients had whole exome sequencing performed at the Leeds Institute of Biomedical and Clinical Sciences (Leeds, UK). Testing methodology, genotype results and systemic phenotypes of both patients have been previously reported by Smith et al. ${ }^{5}$ 
Both patients had a full ophthalmic examination by an ocular genetic specialist $(A V L)$, including visual acuity, slit lamp and dilated fundus examination. Fundus autofluorescence (FAF) images, Goldmann visual fields and spectral domain-optical coherence tomography (SD-OCT) images were obtained. Full-field electroretinogram (ffERG) and multifocal electroretinogram (mfERG) were performed in accordance with the International Society of Clinical Electrophysiology and Vision standards. ${ }^{6}$

\section{Results:}

The BCVA, anterior segment examination, fundus examination, color vision and cycloplegic refraction for each patient are summarized in Table 1.

Case 1: This 12-year-old boy with congenital high frequency sensorineural hearing loss (SNHL) was the product of an uncomplicated pregnancy. Family history was unremarkable. Initially he presented with delayed speech which resulted in the diagnosis of SNHL at 18 months old, requiring hearing aids. He had positional posterior plagiocephaly, toe walking and learning disability with normal IQ testing and a normal brain MRI scan. Hyperpigmentation was observed along the lines of Blaschko on the left shoulder and arm, together with Beau's lines on both great toe nails (Figure1A), and amelogenesis imperfecta on the secondary teeth. Between 5 and 9 years of age, he had occlusion therapy for strabismic amblyopia of his right eye. Pigmentary retinal changes were noted at 3 years old. He had no nyctalopia or evidence of constricted visual fields. He had continued ophthalmic follow up and subsequently was referred to Wills Eye Hospital at age 12 for further evaluation.

Fundus autofluorescence (FAF) and optical coherence tomography (OCT) images are shown in Figure 2. The patient did not have intravenous fluorescein angiography testing. 
The mfERG showed decreased central and paracentral amplitudes and delayed implicit time in both eyes (Figure2). The ffERG waveforms were normal in response to scotopic and combined flash stimuli in both eyes, but showed decreased amplitude and delayed implicit time of the A- and B-wave in response to single flash photopic stimuli and $30 \mathrm{~Hz}$ flicker (Figure 2). Goldmann visual fields were normal in both eyes. The patient was treated with topical and oral acetazolamide for his intraretinal cystoid spaces and had improvement from $20 / 125$ to $20 / 80$ in the right eye and $20 / 40$ to $20 / 25$ in the left eye. After 5 years of follow up, the patient's visual acuity was stable with oral acetazolamide as his only medication.

Karyotype revealed a normal male karyotype. A single nucleotide polymorphism (SNP) microarray (Quest Diagnostic Nichols Institute, Chantilly, Virginia) found a 146-kb deletion at $5 q 21.3$ and $466 \mathrm{~kb}$ duplication at 1p32.1p31.3, both of which were maternally inherited. Maternal eye examination status post LASIK surgery showed a mildly shallow anterior chamber, bilateral cortical lens flecks, a normal fundus and a normal appearance with optical coherence tomography. Paternal eye examination showed high myopic astigmatism with bilateral cortical lens flecks and a normal fundus. Sequencing of the exons and flanking intronic areas of CNNM4 and NR2E3 (Casey Eye Institute, Portland, OR, USA) detected no abnormal findings and no deletions or duplications were found. The OtoGenome hearing loss panel of 71 genes (Laboratory for Molecular Medicine, Cambridge, MA, USA) was normal. Whole exome sequencing revealed compound heterozygosity in the PEX6 gene: a novel mutation c.275T>G (p.Val92Gly) and known mutation c.1802G>A (p.Arg601Gln). ${ }^{2}$ The novel mutation affects a region of the gene that is conserved in mammalian species. ${ }^{5}$ Both SIFT and Mutation-Taster programs predict pathogenicity. PolyPhen-2 predicts the change to be probably damaging. The known mutation c.1802G $>\mathrm{A}$ 
is maternally inherited and the novel mutation c. $275 T>G$ is paternally inherited. This patient is Family2 in Smith et al. ${ }^{5}$

Case 2: This 7 year- old girl is the product of an uncomplicated pregnancy born to a 33-year-old mother, who has a periauricular skin tag, hypoglossal duct surgery and hypothyroidism, and a healthy 36 -year-old father. There is no known consanguinity. Family history is otherwise unremarkable except for the patient's brother who had hydronephrosis requiring bilateral ureteral implantation surgery. Our patient had a normal voiding cystourethrogram (VCUG) in the neonatal period.

She was entirely well until 3 years old when she was diagnosed with bilateral sensorineural hearing loss, requiring hearing aids, with normal speech (Figure 1B). She had amelogenesis imperfecta of her primary and secondary molars (Figure 1C). Nails were normal. She had a small cutaneous capillary hemangioma on her right lower chest. Between 3 and 5 years old, she was treated for amblyopia in her left eye with patching and glasses. At 7 years old, retinal pigmentary changes were found. Her night vision was normal, but she sometimes had photophobia. She was referred to Wills Eye Hospital for further evaluation.

FAF and OCT images are shown in Figure 3. FfERG waveforms were normal in response to scotopic stimuli, photopic stimuli, combined flash and $30 \mathrm{~Hz}$ flicker (Figure 3). MfERG waveforms were decreased in amplitude paracentrally in response to pseudorandom honeycomb pattern stimuli in both eyes (Figure 3). The responses were slightly better centrally and peripherally. Goldmann visual fields were normal.

SNP Microarray analysis (Quest Diagnostic Nichols Institute, Chantilly, VA, USA) was normal. OtoChip screening (Molecular Otolaryngology and Renal Research Laboratories, IA, 
USA) was negative. Sequencing of the exons and flanking intronic areas of CNNM4 (Casey Eye Institute, Portland, OR, USA) was normal and no deletions or duplications were found. Whole exome sequencing revealed compound heterozygosity for mutations in PEX6, these being the known mutation ${ }^{2} \mathrm{c} .1802 \mathrm{G}>\mathrm{A}(\mathrm{p} . \mathrm{Arg} 601 \mathrm{GIn})$ mutation and a novel missense mutation c.296G>T (p.Arg99Leu). The novel mutation affects a region of the gene that is conserved in mammalian species. ${ }^{5}$ The Mutation-Taster program predicts pathogenicity. PolyPhen-2 predicts the change to be probably damaging while SIFT predicts this variation to be non-deleterious. The known mutation c. $1802 \mathrm{G}>\mathrm{A}$ is maternally inherited and the novel mutation c. $296 \mathrm{G}>\mathrm{T}$ is paternally inherited. This patient is Family 3 from Smith et al. ${ }^{5}$

\section{Discussion:}

The ocular manifestations of Heimler syndrome were first reported by Lima et al., who characterized the macular dystrophy in a 29-year-old female, later shown to be compound heterozygous for mutations in $P E X 1^{4}$. Key findings included decreased visual acuity with "salt and pepper" fundus, hyper and hypoautofluorescent dots in the posterior pole and periphery of the retina, loss of the "inner/outer segment boundary" and RPE thinning in OCT with normal ffERG. Ratbi et al. described "retinal pigmentation", macular dystrophy and visual impairment, and more recently, retinitis pigmentosa. Smith et al. described 6 families with Heimler syndrome. Five had initially been misdiagnosed as Usher syndrome. The ocular findings were briefly described in one patient with PEX6 mutations who is our patient 2. Families 2 and 3 described by Smith et al. are our patients 1 and 2 , respectively. ${ }^{2}$ We herein report further detailed characterization of their ocular phenotype. To the best of our knowledge, our cases represent the first report of detailed characterization of the ocular findings in Heimler syndrome due to mutations in PEX6. 
Heimler syndrome exhibits phenotypic variability. SNHL can be unilateral or bilateral and nails can be normal. ${ }^{7-8}$ Amelogenesis imperfecta usually manifests in secondary teeth, with posterior (premolar and molar) teeth more severely affected than anterior (incisor) teeth. Amelogenesis imperfecta is also seen in Jalili syndrome,${ }^{9}$ hyperbilirubinemia, premature birth, hypoparathyroidism, viral infections and in response to excessive fluoride or tetracycline. Jalili syndrome is associated with cone-rod dystrophy and is therefore relevant to the differential diagnosis in our patient, although our patient did not have mutations in CNNM4.

Our case 1 had typical findings of Heimler syndrome including SNHL, amelogenesis imperfecta and abnormal nails. He also had posterior plagiocephaly, toe walking and learning disability. Posterior plagiocephaly without lambdoidal synostosis is frequent in the general population when positional and is likely unrelated. SNP microarray revealed a 146$\mathrm{kb}$ deletion at $15 q 21.3$ and $466 \mathrm{~kb}$ duplication at $1 p 32.1 p 31.3$, both inherited from his unaffected mother, suggesting that these copy number variations are likely benign. Sequencing of CNNM4 was negative and screening with the OtoGenome hearing loss panel was normal. Delayed development with amelogenesis imperfecta had been reported with mutations in PEX6. ${ }^{5}$ Patient 2 also had findings typical of Heimler syndrome, including SNHL and amelogenesis imperfecta. SNP microarray analysis, CNNM4 sequencing and deletion/duplication analysis were normal. Whole exome sequencing revealed PEX6 pathogenic sequence changes in both patients. ${ }^{5}$

The discovery that hypomorphic PEX1 and PEX6 mutations cause Heimler syndrome $e^{2,5}$ identifies the condition as a peroxisomal disorder. One study suggests that mutations in PEX1 are more common ${ }^{2}$ while another found PEX6 mutations to be more 
common. ${ }^{5}$ Other peroxisomal disorders include Zellweger syndrome, neonatal adrenoleukodystrophy (NALD), infantile Refsum disease (IRD) and chondrodysplasia punctata. Mutations in PEX1 and PEX6 may also result in a form of Zellweger syndrome, which is characterized by craniofacial abnormalities, neurologic dysfunction, and liver dysfunction. ${ }^{10-11}$ Ocular manifestations were not specifically described. In other forms of Zellweger syndrome, some of which were not molecularly defined, ocular findings include nystagmus, cloudy corneas, glaucoma, cataract, retinal dystrophy with extinguished ERG, macular intraretinal cystoid spaces and optic atrophy. ${ }^{10,12-18}$ Our patients, and those reported by Ratbi and Lima, had a milder form of retinal dystrophy compared to other patients with Zellweger syndrome.

The peroxisome is a cytoplasmic organelle. Its main function is the breakdown of very long chain fatty acids through beta-oxidation. The PEX1 and PEX6 proteins bind with adenosine triphosphate (ATP) to form a heterohexameric ATPase which is associated with various cellular activities that fuel essential protein transport across peroxisomal membranes, the final steps of peroxisomal matrix-protein import. ${ }^{19-23}$ PEX1 and PEX6 are expressed in the retina, especially in photoreceptors. ${ }^{24}$ Abnormal PEX6 and PEX1 proteins result in abnormal peroxisomal function, leading to the accumulation of very long chain fatty acids. Histopathology of other peroxisomal disorders shows accumulation of characteristic bileaflet fatty acid inclusions in photoreceptors, RPE and pigment laden macrophages. ${ }^{13}$ Fatty acid accumulation may create the lipofuscin-like substances which appear as hyperfluorescent flecks seen on FAF in our patients.

Our patients showed similarities to and differences from the ocular manifestations resulting from the mutations in PEX1. Visual acuity in our case 1 and Lima's patients showed 
asymmetrical visual loss, while patient 2 had BCVA of 20/20 in both eyes. The decreased vision in the right eye in patient 1 may be attributed, in part, to strabismic amblyopia or the macular cysts, or a combination of both. Anterior segments were normal, except our case 1 which had mild cortical cataract, which has not previously been reported in Heimler syndrome. OCT revealed intraretinal cystoid spaces in the outer plexiform layer in our case 1, Lima's patient and Ratbi's patient. We found mottling of the macular RPE and hyperfluorescent deposits with central macular sparing by FAF in our patients as also reported by Lima et al.

FfERGs were normal in patient2 and showed mild photopic abnormalities in patient1 which differs from Ratbi's patient, who harbored PEX1 mutations and showed abnormal scotopic and photopic responses. The mfERG in our patients are abnormal, consistent with the observed macular dystrophy as also reported by Lima. ${ }^{4}$ The patient of Lima et al. was more severely affected. This may be due to the patient's older age ( 29 years old) or may perhaps suggest a more potent effect of PEX1 mutations. The patient of Ratbi et al., had a retinitis pigmentosa phenotype not seen in our patients. We had personal communication with the physician of the patients from family 5 in Smith et al. ${ }^{5} \mathrm{~A}$ pair of siblings with Heimler syndrome who harbored PEX6 mutation, they had a retinitis pigmentosa phenotype with intraretinal cystoid spaces similar to Ratbi's patient. ${ }^{3}$ Our ERG findings suggest a predominant effect of the PEX6 mutation on the cone system which may be due to fatty acid deposition in macula as shown by FAF.

The intraretinal cystoid spaces in our case 1 may or may not represent cystoid macular edema. IVFA had not been done to distinguish these possibilities. The patient was treated with topical and oral acetazolamide and had some improvement of visual acuity and 
remained stable after 5 years follow-up with oral acetazolamide as his maintenance medication. The patient reported by Lima et al. showed non-leaking intraretinal cystoid spaces by IVFA. Treatment details were not provided. Ratbi's patient, who had been treated with oral acetazolamide, intravitreal triamcinolone acetonide and intravitreal ranibizumab, did not show resolution of intraretinal cystoid spaces. The OCTs of our patients were variable and not particularly correlated with their vision as previously reported..$^{25}$

In conclusion, we report the ocular findings of Heimler syndrome due to PEX6 mutations. Ophthalmic examination can be helpful in this disorder, showing a characteristic pattern on FAF along with changes on OCT, ffERG, and mfERG. Patients with intraretinal cystoid spaces may benefit from carbonic anhydrase therapy. Long term follow-up is required to better understand the ocular prognosis of this disease. Heimler syndrome, and ocular examination with diagnostic testing, should be considered in patients with SNHL or amelogenesis imperfecta.

\section{Declaration of interest}

This work was supported in part by the Foerderer Fund (AVL), The Robison D. Harley, MD Endowed Chair in Pediatric Ophthalmology and Ocular Genetics (AVL) and RP Fighting Blindness and Fight For Sight (the RP Genome Project GR586 - CI). Neither the sponsor nor the funding organizations had a role in the design or conduct of this research. 


\section{References}

1. Heimler A, Fox JE, Hershey JE, Crespi P. Sensorineural hearing loss, enamel hypoplasia, and nail abnormalities in sibs. Am J Med Genet 1991;39:192-95.

2. Ratbi I, Falkenberg KD, Sommen M, Al-Sheqaih N, Guaoua S, Vandeweyer G, Urquhart JE, Chandler KE, Williams SG, Roberts NA, et al. Heimler syndrome is caused by hypomorphic mutations in the peroxisome-biogenesis genes PEX1 and PEX6. Am J Hum Genet 2015;97:535-45.

3. Ratbi I, Jaouad IC, Elorch H, Al-Sheqaih N, Elalloussi M, Lyahyai J, Berraho A, Newman WG, Sefiani A. Severe early onset retinitis pigmentosa in Moroccan patient with Heimler syndrome due to novel homozygous mutation of PEX1 gene. Eur J Med Genet 2016;59:507-11.

4. Lima LH, Barbazetto IA, Chen R, Yannuzzi LA, Tsang SH, Spaide RF. Macular dystrophy in Heimler syndrome. Ophthalmic Genet 2011;32:97-100.

5. Smith CEL, Poulter JA, Levin AV, Capasso JE, Price S, Ben-Yosef T, Sharony R, Newman WG, Shore RC, Brookes SJ, et al. Spectrum of PEX1 and PEX6 variants in Heimler syndrome. Eur J Hum Genet Public Online First 15 June 2016.

doi:10.1038/ejhg.2016.62.

6. McCulloch LD, Marmor MFM, Brigell MG, Hamilton R, Holder GE, Tzekov R, Bach M. ISCEV standard for full-field clinical electroretinography (2015 update). Doc Ophthalmol 2015;130:1-12.

7. Tischkowitz M, Clenaghan C, Davies S, Hunter L, Potts J, Verhoef S. Amelogenesis imperfecta, sensorineural hearing loss, and Beau's lines, a second case report of Heimler's syndrome. J Med Genet 1999;36:941-43. 
8. Pollak C, Floy M, Say B. Sensorineural hearing loss and enamel hypoplasia with subtle nail findings: another family with Heimler's syndrome. Clin Dysmorphol 2003;12:5558.

9. Parry DA, Mighell AJ, El-Sayed W, Shore RC, Jalili IK, Dollfus H, Bloch-Zupan A, Carlos R, Carr IM, Downey LM, et al. Mutations in CNNM4 cause Jalili syndrome, consisting of autosomal-recessive cone-rod dystrophy and amelogenesis imperfecta. Am J Hum Genet 2009;84(2):266-73.

10. Rosewich H, Ohlenbusch A, Gartner J. Genetic and clinical aspects of Zellweger spectrum patients with PEX1 mutations. J Med Genet 2005;42:e58.

11. Cho SY, Chang YP, Park JY, Park HD, Sohn YB, Park SW, Kim SH, Ji S, Kim SJ, Choi EW, et al. Two novel PEX1 mutations in a patient with Zellweger syndrome: the first Korean case confirmed by biochemical and molecular evidence. Ann Clin Lab Sci 2011;41:182-87.

12. Levesque S, Morin C, Guay SP, Villeneuve J, Marquis M, Yik WY, Jiralerspong S, Bouchard L, Steinberg S, Hacia JG, et al. A founder mutation in the PEX6 gene is responsible for increased incidence of Zellweger syndrome in a French Canadian population. BMC Med Genet 2012;13:72.

13. Folz SJ, Trobe JD. The peroxisome and the eye. Surv Ophthalmol 1991;35:353-68.

14. Al-Essa M, Dhaunsi GS, Rashed M. Zellweger syndrome in Saudi Arabia and its distinct features. Clin Pediatr (Phila) 1999;38:77-86.

15. Stanesu-Segal B, Evrard P. Zellweger syndrome, retinal involvement. Metab Pediatr Syst Ophthalmol. (1985) 1989;12(4):96-99. 
16. Lyons CJ, Castano G, McCormick AQ, Applegarth D. Leopard spot retinal pigmentation in infancy indicating a peroxisomal disorder. Br J Ophthalmol 2004;88:191-92.

17. Cohen SM, Green WR, de la Cruz ZC, Brown FR 3rd, Moser HW, Luckenbach MW, Dove DJ, Maumenee IH. Ocular histopathologic studies of neonatal and childhood adrenoleukodystrophy. Am J Ophthalmol 1983;95:82-96.

18. Witters P, Régal L, Waterham HR, De Meirleir L, Wanders RJ, Morava E. Normal cognitive outcome in a PEX6 deficient girl despite neonatal multisystem presentation. Am J Med Genet 2016;170:1642-46.

19. Raas-Rothschild A, Wanders RJ, Mooijer PA, Gootjes J, Waterham HR, Gutman A, Suzuki Y, Shimozawa N, Kondo N, Eshel G, et al. A PEX6-defective peroxisomal biogenesis disorder with severe phenotype in an infant, versus mild phenotype resembling Usher syndrome in the affected parents. Am J Hum Genet 2002;70:106268.

20. Seo JG, Lai CY, Miceli MV, Jazwinski SM. A novel role of peroxin PEX6: suppression of aging defects in mitochondria. Aging Cell 2007;6:405-13.

21. Tan D, Blok NB, Rapoport TA, Walz T. Structures of the doublering AAA ATPase PEX1PEX6 involved in peroxisome biogenesis. Febs J 2016;283:986-92.

22. Ciniawsky S, Grimm I, Saffian D, Girzalsky W, Erdmann R, Wendler P. et al. Molecular snapshots of the Pex1/6 AAA+ complex in action. Nat Common 2015;6:7331.

23. Gardner BM, Chowdhury S, Lander GC, Martin A. The Pex1/Pex6 complex is a heterohexameric AAA+ motor with alternating and highly coordinated subunits. J Mol Biol 2015;427:1375-88. 
24. Zaki MS, Heller R, Thoenes M, Nürnberg G, Stern-Schneider G, Nürnberg P, Karnati S, Swan D, Fateen E, Nagel-Wolfrum K, et al. PEX6 is expressed in photreceptor Cilia and mutated in deafblindness with enamel dysplasia and microcephaly. Hum Mutat 2016;37(2):170-74.

25. Lingao MD, Ganesh A, Karthikeyan AS, Al Zuhaibi S, Al-Hosni A, Al Khayat A, Capasso J, Trumler AA, Stroh E, Al Shekaili H, et al. Macular cystoid spaces in patients with retinal dystrophy. Ophthalmic Genet 2016;37:377-83. 